\title{
Magnetic field reversals and long-time memory in conducting flows
}

\author{
P. Dmitruk,${ }^{1}$ P. D. Mininni, ${ }^{1}$ A. Pouquet, ${ }^{2}$ S. Servidio, ${ }^{3}$ and W. H. Matthaeus ${ }^{4}$ \\ ${ }^{1}$ Departamento de Física, Facultad de Ciencias Exactas y Naturales, Universidad de Buenos Aires and IFIBA, \\ CONICET, Buenos Aires, Argentina \\ ${ }^{2}$ Department of Atmospheric and Space Physics, University of Colorado and National Center for Atmospheric Research, \\ Boulder, Colorado, USA \\ ${ }^{3}$ Dipartimento di Fisica, Universita della Calabria, Cosenza, Italy \\ ${ }^{4}$ Bartol Research Institute and Department of Physics and Astronomy, University of Delaware, Newark, Delaware, USA
}

(Received 10 December 2013; revised manuscript received 17 July 2014; published 16 October 2014)

\begin{abstract}
Employing a simple ideal magnetohydrodynamic model in spherical geometry, we show that the presence of either rotation or finite magnetic helicity is sufficient to induce dynamical reversals of the magnetic dipole moment. The statistical character of the model is similar to that of terrestrial magnetic field reversals, with the similarity being stronger when rotation is present. The connection between long-time correlations, $1 / f$ noise, and statistics of reversals is supported, consistent with earlier suggestions.
\end{abstract}

DOI: 10.1103/PhysRevE.90.043010

PACS number(s): 47.65.-d, 47.27.E-, 47.35.Tv, 91.25.Mf

\section{INTRODUCTION}

The origin of magnetic field reversals in Earth's magnetic field is a matter of debate. Reversals take place rapidly, within a scale of $\sim 1000$ years, but infrequently, distanced apart by periods of $10^{4}-10^{7}$ years $[1,2]$.

Reversals, first thought to be a purely random process, are now known to display long-term memory with deviations from a purely Poisson process [3]. In many systems, such a high degree of variability may be associated with features of the power spectrum of the time series known as " $1 / f$ " noise, an indication of the presence of correlations over a wide range of time scales [4-7]. $1 / f$ signals are found in many physical systems [8,9], including the intensity of the geomagnetic field $[10,11]$. We examine this phenomenon by employing a simple model consisting of incompressible nondissipative magnetohydrodynamics (MHD) in spherical geometry. The results demonstrate the presence of reversals possessing $1 / f$ noise where rotation and/or magnetic helicity play important roles. Remarkably, the distribution of waiting times between reversals follows a power law that is comparable to the record of terrestrial magnetic reversals.

Earth's magnetic field is sustained by a dynamo process: Motions of the conducting fluid core generate and sustain magnetic fields against Ohmic dissipation. Although MHD contains the basic physics of the dynamo, the complete terrestrial problem requires solving either compressible, Boussinesq, or anelastic MHD equations for the velocity, the magnetic field, and the temperature, in a spherical shell with a possible inner solid conducting core, and surrounded by a mantle [12,13]. Additional realism requires more complexity in chemistry, equations of state, and boundary conditions. Even with advanced supercomputers, only few reversals can be simulated [12-14], and studies of the long-time statistics of reversals are therefore out of reach.

Experiments reproducing dynamos in laboratory turbulent flows display magnetic field reversals $[15,16], 1 / f$ noise, and long-term memory. Still, a theoretical understanding of these features remains incomplete since the origin of correlations with time scales much greater than the characteristic nonlinear time associated with the largest eddies in the system is unknown.
Many physical causes have been considered to explain the origin and statistics of the reversals, including the effect of tides, departures of the mantle from spherical geometry, or low magnetic Reynolds number effects. We show that the MHD equations in their simplest nonlinear form (incompressible and ideal) in a simple geometry (spherical surrounded by a perfect conductor) already include the ingredients required for magnetic field reversals, long-time correlations, $1 / f$ noise, and non-Poisson statistics compatible with that observed in the geodynamo.

\section{MODEL}

The ideal MHD equations are solved using a spectral method that preserves to numerical accuracy all ideal quadratic invariants with no numerical dissipation or dispersion. For very long-time integrations, this is the only method that ensures adequate conservation. Since the initial energy introduced in the system is conserved, no external forces are needed to sustain the velocity and magnetic fields. For a purely spectral Galerkin method, we use spherical Chandrasekhar-Kendall functions as a basis, expanding the fields in spectral space $[17,18]$. A fourth-order Runge-Kutta method is used to evolve the system in time.

With the magnetic field confined in the interior of the sphere, the system has two quadratic conserved quantities: the total energy (kinetic plus magnetic, $E=\frac{1}{2} \int|\mathbf{v}|^{2}+|\mathbf{b}|^{2} d V$, with $\mathbf{v}, \mathbf{b}$ the velocity and magnetic fields) and the magnetic helicity $\left(H_{m}=\int \mathbf{a} \cdot \mathbf{b} d V\right.$, a measure of linkage or handedness of the magnetic field, with $\mathbf{a}$ the vector potential, $\nabla \times \mathbf{a}=\mathbf{b}$ ). $E$ is transferred towards small scales ("direct cascade"), while $H_{m}$ is transferred towards large scales ("inverse cascade"). In the ideal system, $H_{m}$ condenses at the largest available scales [19]. In our simulations, long-time-scale correlations arise when $H_{m}$ is nonzero. Long-time correlations also arise due to symmetry breaking by rotation [20]. Here we show for the rotating sphere that the magnetic dipole moment reverses with respect to the rotation direction, displaying $1 / f$ noise and long-term memory even when the magnetic helicity is zero. A recent related study [21] reported persistence of the magnetic dipole associated with broken ergodicity effects [22]. Broken ergodicity of fluid systems may also be viewed as 
"delayed ergodicity" in which very long-time correlations delay ergodically covering the phase space [23].

The incompressible ideal MHD equations solved for the evolution of the velocity field $\mathbf{v}$ and magnetic field $\mathbf{b}$ (in Alfvenic units) are

$$
\begin{gathered}
\frac{\partial \mathbf{v}}{\partial t}=\mathbf{v} \times \boldsymbol{\omega}+\mathbf{j} \times \mathbf{b}-\nabla\left(\mathcal{P}+\frac{v^{2}}{2}\right)-2 \boldsymbol{\Omega} \times \mathbf{v}, \\
\frac{\partial \mathbf{b}}{\partial t}=\nabla \times(\mathbf{v} \times \mathbf{b}),
\end{gathered}
$$

for vorticity $\boldsymbol{\omega}=\nabla \times \mathbf{v}$; electric current density $\mathbf{j}=\nabla \times \mathbf{b}$; normalized pressure $\mathcal{P}$; and rotation rate $\boldsymbol{\Omega}$. The units are normalized to the spherical radius $R$ and initial root mean square velocity $v_{0}=\left\langle\mathbf{v}^{2}\right\rangle^{1 / 2}$, so $R=1$ and $v_{0}=1$, and the time unit is $t_{0}=R / v_{0}=1$ (later time scales are rescaled to $\mathrm{Ma}=10^{6}$ years, based on the longest observed waiting time between reversals). We consider vanishing normal velocity and magnetic field components at the sphere boundary. For the simulations, 980 coupled Chandrasekhar-Kendall (C-K) modes are followed in time. The $\mathrm{C}-\mathrm{K}$ functions are

$$
\mathbf{J}_{i}=\lambda \nabla \times \mathbf{r} \psi_{i}+\nabla \times\left(\nabla \times \mathbf{r} \psi_{i}\right),
$$

where we work with a set of spherical orthonormal unit vectors $(\hat{r}, \hat{\theta}, \hat{\phi})$, and the scalar function $\psi_{i}$ is a solution of the Helmholtz equation, $\left(\nabla^{2}+\lambda^{2}\right) \psi_{i}=0$. The explicit form of $\psi_{i}$ is

$$
\psi_{i}(r, \theta, \phi)=C_{q l} j_{l}\left(\left|\lambda_{q l}\right| r\right) Y_{l m}(\theta, \phi),
$$

where $j_{l}\left(\left|\lambda_{q l}\right| r\right)$ is the order- $l$ spherical Bessel function of the first kind, $\left\{\lambda_{q l}\right\}$ are the roots of $j_{l}$ indexed by $q$ (so that the function vanishes at $r=1)$, and $Y_{l m}(\theta, \phi)$ is a spherical harmonic in the polar angle $\theta$ and the azimuthal angle $\phi$. The subindex $i$ is a shorthand notation for the three indices $(q, l, m) ; q=1,2,3, \ldots$ corresponds to the positive values of $\lambda$, and $q=-1,-2,-3, \ldots$ indexes the negative values; finally $l=1,2,3, \ldots$, and $-l \leqslant m \leqslant l$. The $\mathrm{C}-\mathrm{K}$ functions satisfy

$$
\nabla \times \mathbf{J}_{i}=\lambda_{i} \mathbf{J}_{i}
$$

With the proper normalization constants, they are a complete orthonormal set. The values of $\left|\lambda_{i}\right|$ play a role similar to the wave number $k$ in a Fourier expansion. Note that the boundary conditions, as well as the Galerkin method to solve the equations inside the sphere using this base, were chosen to ensure conservation of all quadratic invariants of the system (total energy and magnetic helicity).

The initially excited modes for the runs are those for $q= \pm 3, l=3$ and all possible values of $m$. With proper initial values for the expansion coefficients of the $\mathrm{C}-\mathrm{K}$ functions, the initial values of the quadratic quantities can be chosen. In all the runs, the initial total energy is set to $E=1$ (dimensionless units). We set the initial magnetic and kinetic energies to $E_{m}=E_{k} \approx 0.5$. The runs with nonzero magnetic helicity have $H_{m} \approx 0.03$. As a comparison, note that for the $q=3, l=3$ mode alone, $H_{m} / E_{m}$ is no more than about 0.072 (this is the maximum value of $\left|H_{m} / E_{m}\right|$ if only modes with $|q|=3$, $l=3$, and one sign of $\lambda$ are excited). So, the chosen value of $H_{m}$ (when is nonzero) corresponds to about $85 \%$ of the maximum helicity in the system.

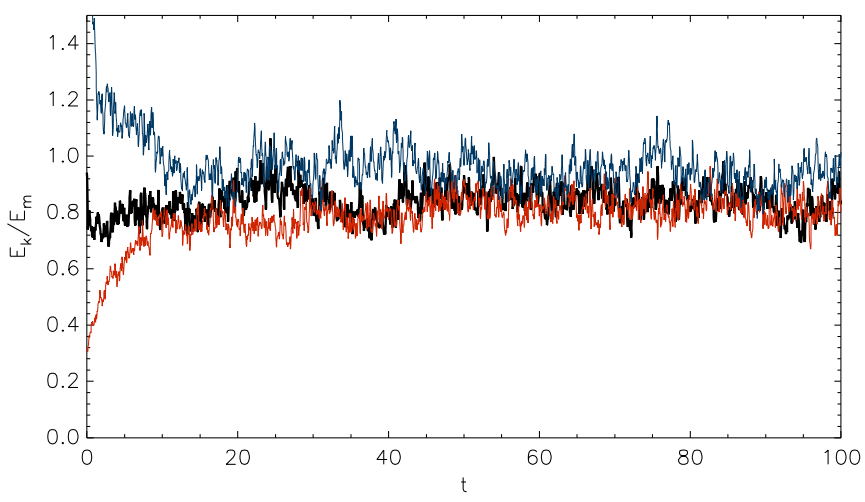

FIG. 1. (Color online) The ratio of kinetic energy vs magnetic energy $E_{k} / E_{m}$ as a function of time, for three runs with different initial conditions and same $H_{m}=0.03, \Omega=16$. Thicker line (black online) $E_{k} / E_{m}(t=0)=1$, intermediate thick line (red online) $E_{k} / E_{m}(t=$ $0)=0.5$, and thin line (blue online) $E_{k} / E_{m}(t=0)=2$.

\section{RESULTS}

\section{A. Kinetic and magnetic energy, field structure}

The values of the total energy $E$ and magnetic helicity remain constant in time (as ideal invariants). The values of the magnetic and kinetic energies $E_{m}$ and $E_{k}$ fluctuate, reaching a statistical steady state after about 20 unit times. The initial ratio of kinetic over magnetic energy is $E_{k} / E_{m}(t=0)=1$ and approaches and fluctuates around $E_{k} / E_{m} \approx 0.9$. We performed two additional runs starting from different initial ratios $E_{k} / E_{m}(0) \approx 2$ and $E_{k} / E_{m}(0) \approx 0.5$, with the same value of magnetic helicity $H_{m}=0.03$. Both cases evolve initially and after about 20 unit times reach the same asymptotical statistical state, with a value of $E_{k} / E_{m} \approx 0.9$. This is shown in Fig. 1 . This asymptotic value corresponds to a steady state with some excess of magnetic energy over kinetic energy which is consistent with the nonzero value of magnetic helicity (which allows condensation at the large scales).

The results about the statistics of the magnetic dipole that follows (next subsection) are not sensitive to the different initial values of the ratio $E_{k} / E_{m}$.

The fields evolve to a highly disordered state, with a wide range of scales present. Figure 2 shows velocity and magnetic field lines for one particular run.

\section{B. Magnetic dipole and statistics of reversals}

We focus on the dynamics of the magnetic dipole moment,

$$
\boldsymbol{\mu}=\frac{1}{2} \int \mathbf{r} \times \mathbf{j} d V
$$

and, in particular, on its $z$ component $\mu_{z}$, which is of importance with rotation $\boldsymbol{\Omega}=\Omega \hat{z}$. We report first results of a run with both nonzero magnetic helicity $\left(H_{m}=0.03\right)$ and nonzero rotation ( $\Omega=16$ in units of $t_{0}^{-1}$ defined above). Figure 3 (top panel) shows the time evolution of the $z$ component of the dipole moment $\mu_{z}$. The simulation extends for $5000 t_{0}$ but for clarity only the segment from $t=50$ to $t=300$ is illustrated. The sign of $\mu_{z}$ changes many times during this period; many reversals are observed. The time 


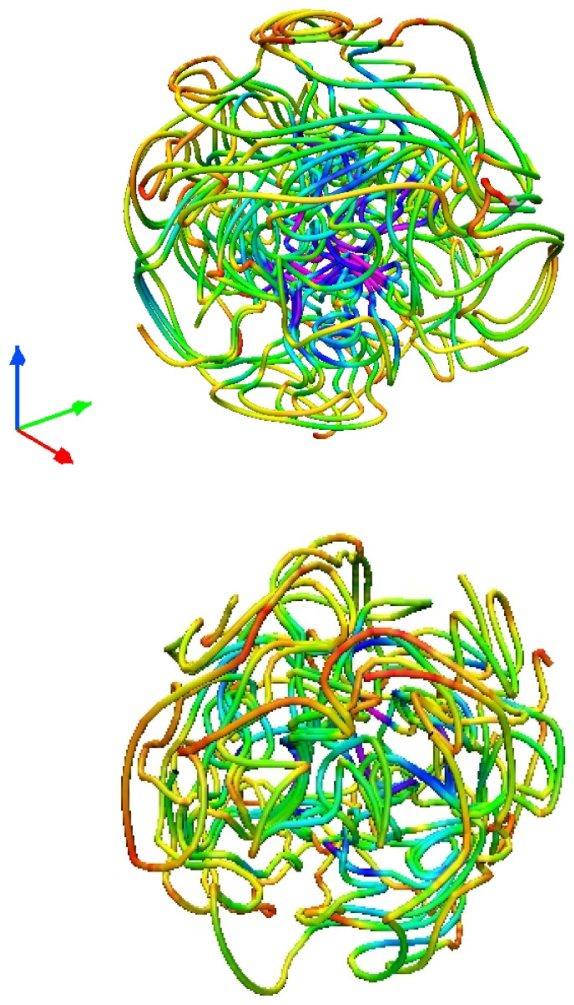

FIG. 2. (Color online) Velocity (top) and magnetic (bottom) field lines in the run with $\Omega=16, H_{m}=0.03$. The field lines change color according to the intensity of the field, from red to yellow, blue, and magenta. The red, green, and blue arrows indicate, respectively, the $x, y, z$ axis, with $\Omega$ in the $z$ direction.

periods between reversals range from short times $(\delta t \sim 1)$ to long times $(\delta t \sim 50)$.

The statistics of these fluctuations are analyzed by computing the frequency power spectrum $P(f)$, shown in Fig. 4 (top). The spectrum is obtained by Fourier transforming the $\mu_{z}(t)$ time series in 10 nonoverlapping samples, averaging the estimates of $P(f)$ to improve statistics. The frequency $f=0.5$ corresponds to the longest nonlinear time scale that can be constructed based on local dimensional arguments, using the longest available scale in the system $2 R$, and a unit root mean square velocity. For a system with no long-term memory effects, $P(f)$ would be flat (constant) at lower frequencies, corresponding to uncorrelated fluctuations at time scales longer than the autocorrelation time $t_{c}=2 R / v_{0}=$ 2. However, the substantial excess power at frequencies $f<1 / t_{c}=0.5$ indicates a long-term memory not controlled by a single correlation time. This effect is known as $1 / f$ noise, corresponding to the typical (approximate) power-law spectrum found at the low frequencies $[5,9]$. A $1 / f$ power law is illustrated in Fig. 4. The appearance of $1 / f$ noise in ideal fluid models has been discussed in [24]. The inset included in Fig. 4 corresponds to the compensated spectrum, that is, $f P(f)$, which should be flat for a $1 / f$ spectrum. This plot indicates clearly the wide range of frequencies for which we can see a $1 / f$ in this case.

To quantify reversals, we compute statistical distribution of times between reversals of $\mu_{z}$, i.e., the waiting time distribution
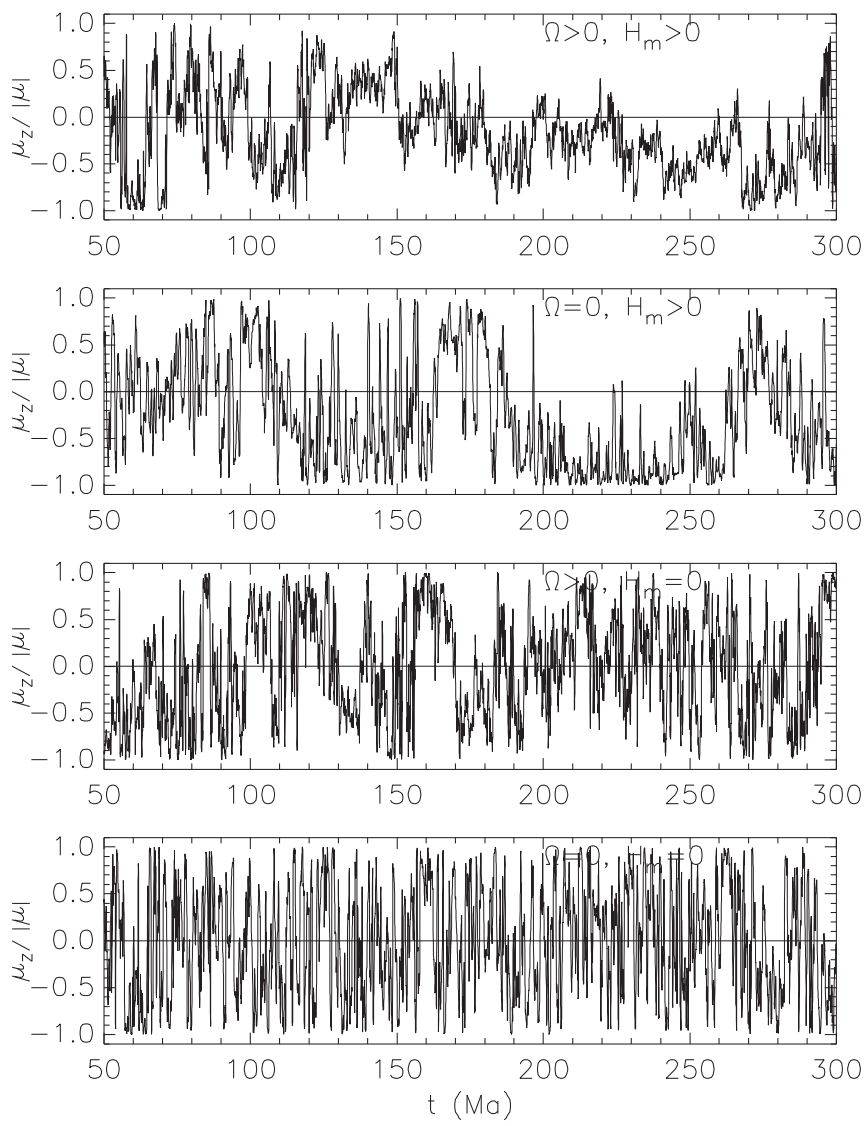

FIG. 3. Time series of the normalized magnetic dipole moment for four different values of the angular velocity of rotation $\boldsymbol{\Omega}$ and magnetic helicity $H_{m}$. Time is measured in units of Ma (mega anni), $1 \mathrm{Ma}=10^{6}$ years, as indicated in the text.

[3]. The probability distribution function (PDF) of the waiting times obtained from the simulation is shown in Fig. 5 (top). Also shown in Fig. 5 is the known distribution of waiting times from data measurements of the geomagnetic reversals in [1]. To compare these, we arbitrarily identify the longest simulation waiting time with the longest reported waiting time for geomagnetic reversals. The latter is $\sim 30 \mathrm{Ma}$. The relevant point here is that the same trend is observed for the waiting times - this corresponds to a power law, indicating the existence of long-term memory and non-Poissonian statistics [3,25]. This long-term memory is associated with the $1 / f$ noise observed in the power frequency spectrum (Fig. 4).

Next, we show results with nonzero magnetic helicity $H_{m}=$ 0.03 but no rotation $(\Omega=0)$. The dipole moment time series, frequency spectrum and waiting time distribution are shown in the second panels of Figs. 3, 4, and 5, respectively. Although the frequency spectrum shows that there is still an excess power at $f<1 / t_{c}$, this effect is weaker than in the case with both rotation and magnetic helicity. The compensated spectrum in the inset of this figure also indicates the range of frequencies for which a $1 / f$ is observed. In addition, the distribution of waiting times departs more from the observational data of Cande and Kent 1995 [1].

Results with zero magnetic helicity $\left(H_{m}=10^{-7}\right)$ and maintaining rotation $(\Omega=16)$ are shown in the third panels of 

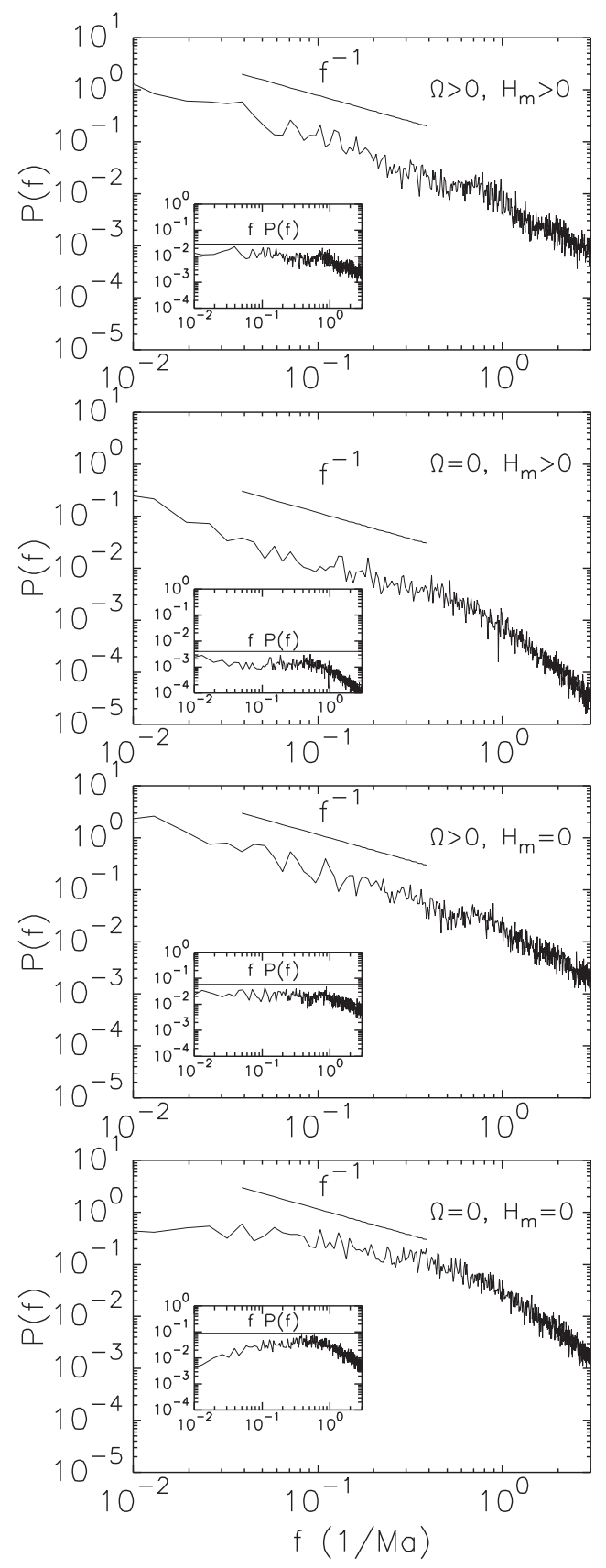

FIG. 4. Frequency spectra of the magnetic dipole moment for four different values of the angular velocity of rotation $\boldsymbol{\Omega}$ and magnetic helicity $H_{m}$. A $f^{-1}$ power-law spectrum is indicated as a reference in each plot. Also, insets show the compensated spectra $f P(f)$ for each case. Units of frequency are $1 / \mathrm{Ma}, 1 \mathrm{Ma}=10^{6}$ years.

Figs. 3, 4, and 5, respectively. These results are similar to the first case of nonzero rotation and magnetic helicity, showing excess power at low frequencies (Fig. 4), flat compensated spectrum (inset), and comparable results with the observational data for the waiting time distribution (Fig. 5).

Finally, we present results with zero magnetic helicity $\left(H_{m}=10^{-7}\right)$ and no rotation $(\Omega=0)$ (bottom panels of Figs. 3, 4, and 5, respectively). In this case, the absence of excess power at lower frequencies is clear. This is more
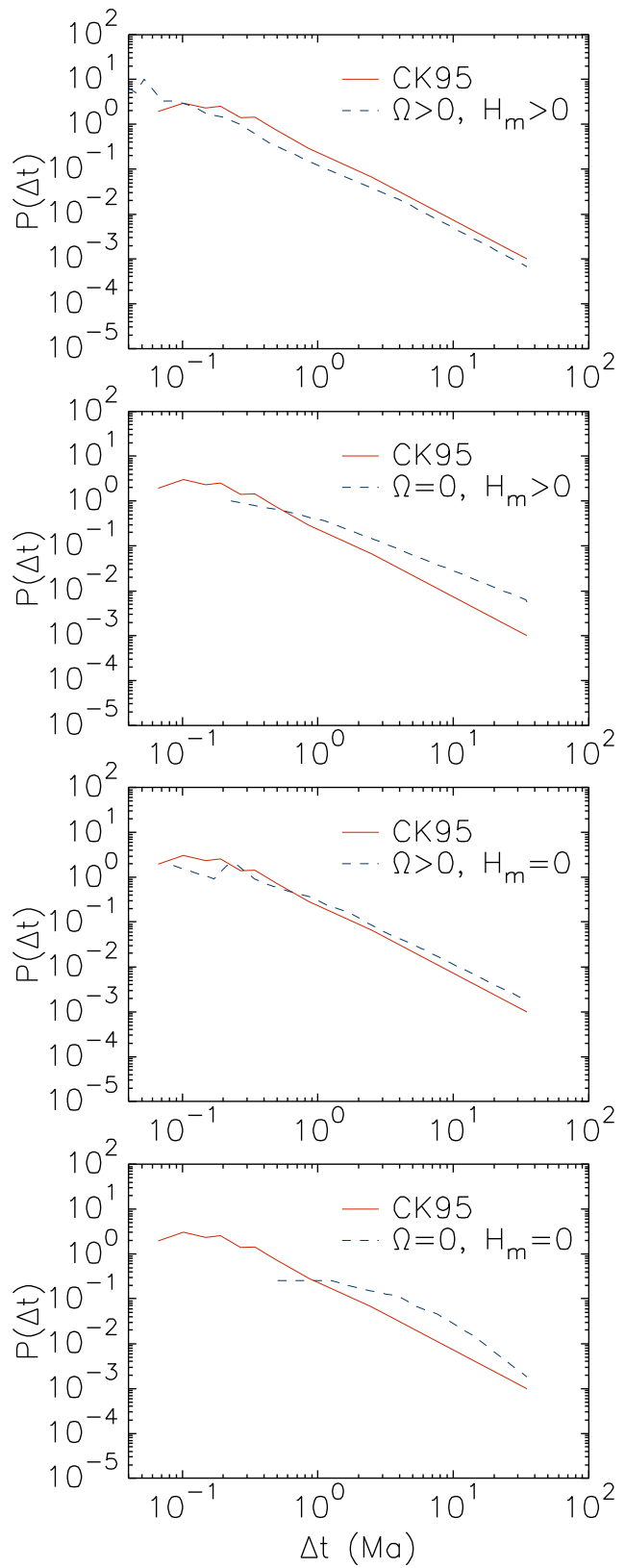

FIG. 5. (Color online) Distribution function of the waiting time between reversals, for four different values of the angular velocity of rotation $\boldsymbol{\Omega}$ and magnetic helicity $H_{m}$ (dashed lines). The continuous (red online) line corresponds to the distribution function for the observational Cande and Kent 1995 data [1].

visible in the compensated spectrum plot (inset). Also, a larger departure from the observational data (Fig. 5) is noted.

\section{The Hurst exponent}

In order to have another measure of comparison we have additionally computed the Hurst exponent $H$ for each of the time series.

The standard definition is that a process $g(t)$ is self-similar, with self-similarity (Hurst) exponent $H \in(0,1)$, if it satisfies

$$
g(\lambda t) \sim \lambda^{H} g(t)(\lambda>0) .
$$




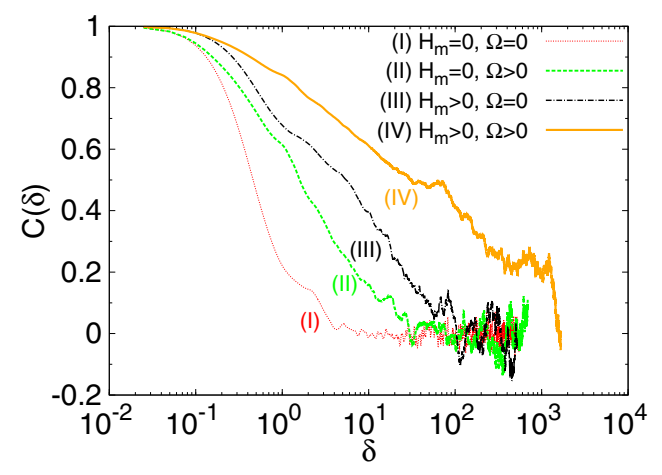

FIG. 6. (Color online) Autocorrelations functions vs time-lag $\delta$.

Even if a process is nonstationary, it can satisfy Eq. (7) if the increment process $\delta g=g(t)-g(t+1)$ is stationary. A canonical example is Brownian motion, for which $H=1 / 2$. When $1>H>0.5$, there are long-range time correlations (persistence), when $0.5>H>0.0$, the series has long-range anticorrelations (antipersistence), and, for a time series with no long-time correlations, $H=0.5$. The $H$ parameter [26,27] is often used to characterize long-range dependencies.

Long-range memory is also connected with power-law decay of the autocorrelation function, with index $\beta$,

$$
C(\delta)=\left\langle\mu_{z}(t) \mu_{z}(t+\delta)\right\rangle \sim \delta^{-\beta},
$$

here written for the magnetic moment $\mu$. The autocorrelation function, for all the cases in our paper is compared in Fig. 6. This indicates clearly the relevance of magnetic helicity and rotation. In fact, where both are present, the tail of the correlation function seems to be more power-law-like.

For cases with long memory, computation of the power spectral density (PSD) becomes difficult; however when the PSD displays a low frequency power-law range, the associated Hurst exponent is also found as

$$
\begin{aligned}
P(f) & \sim f^{-\alpha}, \\
1<\alpha & =2 H+1<3 .
\end{aligned}
$$

In principle, the above can be used to estimate $H$. Performing a fit of the power spectra at low frequencies, we obtained the spectral indexes $\alpha$ for each case, reported in Table I. A different method, described below, is used to obtain $H$.

The main technique we use is based on a structure function analysis in which the expected size of changes for time lag $\delta$ are related to the exponent $H$. For the $q^{\text {th }}$ order structure

TABLE I. Hurst analysis of the dipole moment for simulations with different $H_{m}$ and $\Omega$. Fit to the power spectrum is $\alpha$ [as in Eq. (9)]. Hurst exponent $H$ is an average over $H_{q}$ in Eq. (10).

\begin{tabular}{cccc}
\hline \hline$H_{m}$ & $\Omega$ & $\alpha$ & $H$ \\
\hline$>0$ & $>0$ & $1.13 \pm 0.06$ & $0.107 \pm 0.007$ \\
$>0$ & $=0$ & $1.1 \pm 0.1$ & $0.127 \pm 0.008$ \\
$=0$ & $>0$ & $0.8 \pm 0.1$ & $0.05 \pm 0.01$ \\
$=0$ & $=0$ & $0.3 \pm 0.1$ & $0.010 \pm 0.004$ \\
\hline \hline
\end{tabular}

function one obtains an independent estimate $H_{q}$, where

$$
S_{q}(\delta)=\left\langle\left|\mu_{z}(t+\delta)-\mu_{z}(t)\right|^{q}\right\rangle \sim \delta^{\zeta(q)} \equiv \delta^{q H_{q}} .
$$

A general statement [28] can be made about the family of exponents: $\zeta(q)$ will be concave, $d^{2} \zeta / d^{2}<0$. If the signal has absolute bounds, it can be shown that $\zeta(q)$ is monotonically nondecreasing [29]. Concavity alone is sufficient to define a hierarchy of exponents $\zeta(q)=q H_{q}$.

The relation between definitions in Eqs. (7), (9), and (10) is not immediate, indeed it has some subtleties, clearly documented in the literature. For a good description of the problem, see, for example, [30,31]. A typical example of the self-similar process is given by the fractional Brownian motion (FBM), which can be regarded as a generalization of the well-known Brownian motion which has $H=1 / 2$. Although the power spectral density is not defined for a nonstationary self-similar process such as FBM, it has been shown that a time-averaged power spectra satisfies the relation in Eq. (9), by means of a time-frequency analysis. An explanation can be found in [31].

Figure 7 shows a Hurst analysis for the run with $H_{m}>0$ and $\Omega>0$. Structure functions, computed for $q=1,2,3,4$, reveal that at large $\delta$ (low frequencies), a self-similar scaling is present. As stated in Eq. (7), this behavior is typical of monofractal signals. Note that the range of scales chosen for the fits are comparable to the range of the $1 / f$ noise in the power spectrum. The higher order structure functions give results consistent with monofractality in that $H_{q}$ is independent of $q$ [see Eq. (10)]. The break point of the large-scale noise is roughly at $\delta \sim 1$, the nonlinear time. Finally, we estimate $H=\frac{1}{4} \sum_{q=1}^{4} H_{q}$, and the results are shown in Table I.

The case $H_{m}=0, \Omega=0$ is very particular, having a flat spectrum, while its Hurst exponent is small and consistent with zero, typical of white noise. On the contrary, both the cases with $\left(H_{m}>0, \Omega>0\right)$, and $\left(H_{m}>0, \Omega=0\right)$ have $H \sim 0.1$, indicating that the signal is antipersistent.

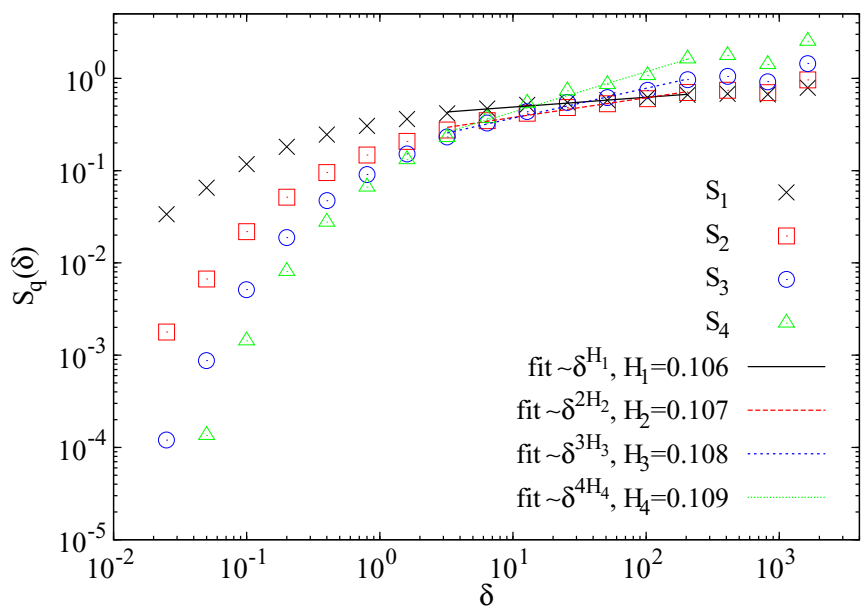

FIG. 7. (Color online) Hurst analysis for $H_{m}>0, \Omega>0$. The structure functions, computed up to the fourth moment, are represented with open symbols, while the fits, from Eq. (10), are reported with lines. In the legend of the plot, the results of the fit $H_{q}$ are reported as well. 


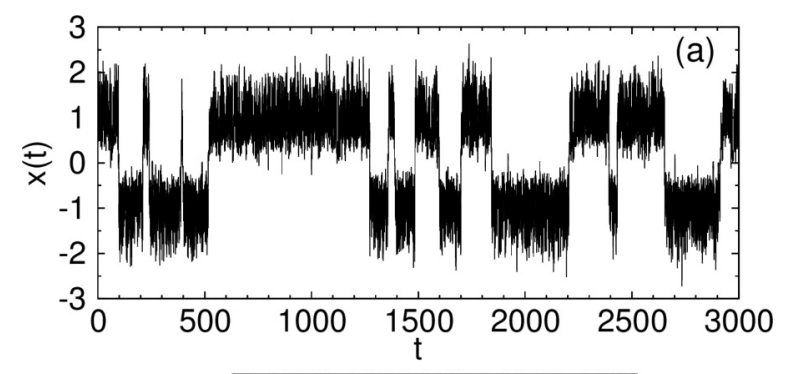

no externally imposed driving. Numerical simulations for durations of 5000 nominal times were performed, for cases including or not including rotation, and for cases including or not including magnetic helicity.

For runs with zero (or very low) helicity and zero rotation, no clear evidence of $1 / f$ noise is found, as the magnetic moments become essentially uncorrelated after about 10 nonlinear times. The waiting time distributions occupy a much narrower span of times, as reversals become numerous. The distribution of waiting times does not match the observational data very well in this case, and appears to form a broad peak around a few nominal times.

When runs are carried out with either rotation or helicity evidence for $1 / f$ noise is found in all cases. In addition, the waiting times begin to resemble the waiting times computed from the CK95 geomagnetic data set. When there is a more distinct spectral signature of $1 / f$ power, one finds a better correspondence of the simulation waiting times and the CK95 waiting times. This is the case for numerical runs in which rotation is present, indicating that this effect is the most important one to be considered to understand the reversals.

In summary, the results from this series of runs show that for reversals to have distributions of waiting times compatible with known observational results it is necessary to have rotation present. Nonzero magnetic helicity is also an asset, but of less significance. For these cases, the magnetic moment dipole has long-time fluctuations, and a frequency analysis shows a $1 / f$-noise-type spectrum. As reported in previous studies, in these cases the turbulence readily generates variability at very long dynamical time scales.

It is of course not possible to draw definite conclusions

At this point we may ask if our Hurst analysis results are comparable to that of geomagnetic reversals. Unfortunately, this analysis cannot be performed on simplified data such as the CK95 data set. To have an independent estimate of $H$ for these systems, we make use of a simple model of geomagnetic dynamo, proposed by Hoyng and Duistermaat (HD) [32]. Very briefly, the HD model, inspired by bistable chaotic systems, describes the axysymmetric component of the dynamo field. The nonlinear evolution takes into account the back-reaction of the Lorentz force on the flow. After simplifications the model reduces to a multidimensional bistable oscillator driven by multiplicative noise [see Eqs. (2)-(4) of [32]]. We solved those model equations numerically, and obtained the time series using the same parameters as in [32]. As reported in Fig. 8 (top), the solution manifests strong similarities with the geomagnetic reversals. The power spectrum (middle panel) exhibits a power law consistent with $1 / f$ noise (slightly steeper.) Finally, the generalized Hurst analysis (bottom) is reported, showing that for this simple geomagnetic model, $H \sim 0.2$. This result is close to the Hurst exponent of the dipole moment derived from our simulations, and suggests that geomagnetic reversals are likely to have $0<H<0.5$, typical of systems with long-range anticorrelations.

\section{CONCLUSIONS}

We obtained reversals of the dipole magnetic field with a simple ideal MHD system, in spherical geometry and about the terrestrial dynamo from an oversimplified model as the present one. Even in the context of the model that we employ, the reported simulations have not been run with parameters as extreme as those found in nature. For example, with eddy speed $1 \times 10^{-8} \mathrm{~km} / \mathrm{s}, R \approx 3000 \mathrm{~km}$, and one rotation per day, a more realistic rotation parameter would be $\Omega t_{0} \sim 10^{5}$. But in that case runs extending to 5000 times $t_{0}$ would correspond to $\sim 10^{9}$ rotations. This would be a discouragingly stiff numerical problem using our accurate (but computationally expensive) Galerkin code. Even then, longer runs, to perhaps $10^{6} t_{0}$ would be required to compute reversals that might occur at $10^{7}$ years. In this perspective, the significance of the present results are largely due to the self-similar character of both the power-law waiting times and the apparently underlying $1 / f$-noise signal. Conceptually, the present results demonstrate that the key physical ingredients present in a simple model of nonlinear magnetohydrodynamics, with rotation, are able to account for statistics of reversals roughly comparable to those observed for the terrestrial dynamo. The requisite long time scales appear to originate in the $1 / f$ noise generated by the model. This $1 / f$-noise generation has been argued previously to be a generic feature of nonlinear systems operating in a regime on which nonlocality of interactions in scale is a prominent feature [7]. As such, we suggest that geomagnetic reversals may in part share their physical origins with a much broader class of nonlinear self-organizing fluid problems. 


\section{ACKNOWLEDGMENTS}

Research supported by Grants No. Proyecto Investigacion Plurianual CONICET0825, No. UBACYT 20020110200359, No. PICT 2011-1529, No. 2011-1626, No. National Science Foundation Atmospheric and Geospace Sciences-1063439, No. Solar Heliospheric and Interplanetary Environment AGS-1156094, and
No. Collaboration in Mathematics and Geophysics/1025183, NASA Solar Probe Plus, Integrated Science Investigation of the Sun, Programa Operativo Regione Calabria Fondo Sociale Europeo 2007/2013, and Marie Curie International Staff Exchange Scheme, Seventh Framework Program-2010-269297 "Turboplasmas."
[1] S. C. Cande and D. V. Kent, J. Geophys. Res. 100, 6093 (1995).

[2] J.-P. Valet, L. Meynadier, and Y. Guyodo, Nature (London) 435 , 802 (2005).

[3] V. Carbone, L. Sorriso-Valvo, A. Vecchio, F. Lepreti, P. Veltri, P. Harabaglia, and I. Guerra, Phys. Rev. Lett. 96, 128501 (2006).

[4] A. VanderZiel, Physica 16, 359 (1950).

[5] S. Machlup, in Sixth International Conference on Noise in Physical Systems (National Bureau of Standards, Washington DC, 1981), p. 157.

[6] E. W. Montroll and M. F. Shlesinger, Proc. Natl. Acad. Sci. USA 79, 3380 (1982).

[7] P. Dmitruk and W. H. Matthaeus, Phys. Rev. E 76, 036305 (2007).

[8] P. Dutta and P. M. Horn, Rev. Mod. Phys. 53, 497 (1981).

[9] B. J. West and M. F. Shlesinger, Int. J. Mod. Phys. B 3, 795 (1989).

[10] L. B. Ziegler and C. G. Constable, Earth Planet. Sci. Lett. 312, 300 (2011).

[11] C. G. Constable and C. Johnson, Phys. Earth Planet. Int. 153, $61(2005)$

[12] G. Glatzmaier and P. Roberts, Nature (London) 377, 203 (1995).

[13] H. Amit, R. Leonhardt, and J. Wicht, Space Sci. Rev. 155, 293 (2010).

[14] P. L. Olson, G. A. Glatzmaier, and R. S. Coe, Earth Planet. Sci. Lett. 304, 168 (2011).

[15] M. Berhanu et al., Europhys. Lett. 77, 59001 (2007).

[16] R. A. Bayliss, C. B. Forest, M. D. Nornberg, E. J. Spence, and P. W. Terry, Phys. Rev. E 75, 026303 (2007).
[17] P. D. Mininni and D. C. Montgomery, Phys. Fluids 18, 116602 (2006).

[18] P. D. Mininni, D. C. Montgomery, and L. Turner, New J. Phys. 9, 303 (2007).

[19] U. Frisch, A. Pouquet, J. Léorat, and A. Mazure, J. Fluid Mech. 68, 769 (1975)

[20] P. D. Mininni, P. Dmitruk, W. H. Matthaeus, and A. Pouquet, Phys. Rev. E 83, 016309 (2011)

[21] J. V. Shebalin, Geophys. Astrophys. Fluid Dyn. 107, 353 (2013).

[22] J. V. Shebalin, Physica D 37, 173 (1989).

[23] S. Servidio, W. H. Matthaeus, and V. Carbone, Phys. Rev. E 78, 046302 (2008).

[24] P. Dmitruk, P. D. Mininni, A. Pouquet, S. Servidio, and W. H. Matthaeus, Phys. Rev. E 83, 066318 (2011).

[25] L. Sorriso-Valvo, F. Stefani, V. Carbone, G. Nigro, F. Lepreti, A. Vecchio, and P. Veltri, Phys. Earth Planet. Int. 164, 197 (2007).

[26] H. E. Hurst, Trans. Am. Soc. Civ. Eng. 116, 770 (1951).

[27] B. B. Mandelbrot and J. R. Wallis, Water Resour. Res. 4, 909 (1968).

[28] A. Davis, A. Mrarshak, W. Wiscombea, and R. Cahalan, J. Geophys. Res. 99, 8055 (1994).

[29] U. Frisch, Proc. R. Soc. London. A 434, 89 (1991).

[30] M. Gilmore, C. X. Yu, T. L. Rhodes, and W. A. Peebles, Phys. Plasmas 9, 1312 (2002).

[31] P. Flandrin, IEEE Transf. Inf. Theory 35, 197 (1989).

[32] P. Hoyng and J. J. Duistermaat, Europhys. Lett. 68, 177 (2004). 